\title{
Fractography as a tool to assess the occurrence of fatigue fractures in complex microstructure structural components
}

\section{Individuazione frattografica di rotture per fatica in componenti metallici con microstrutture complesse}

\author{
D. Firrao, P. Matteis \\ Politenico di Torino - Dipartimento di Scienza Applicata e Tecnologia (DISAT), Corso Duca degli Abruzzi 24, 10129 Torino, \\ Italy \\ donato.firrao@polito.it
}

\begin{abstract}
Fractographic assessment of fatigue fractures may be difficult if they occur in metallic components characterized by low ductility complex microstructures. In these cases reconciliation of known fatigue rupture mechanisms with fractographic appearance of fatigue fractured surfaces is challenging. Special techniques coupled with theory development may be necessary. Pearlitic steels or steels with predominant pearlitic microstructures are among the ones that are visited and their fatigue fractures interpreted. Analogously, fatigued $\mathrm{Al}$ foundry alloys, with hypoeutectic Al-Si compositions, are also illustrated.

SOMMARIO. L'individuazione frattografica delle fratture per fatica risulta spesso piuttosto complessa nei materiali che non esibiscono adeguata duttilità. Microstrutture complesse caratterizzate dalla presenza di numerosi componenti metallografici esibiscono morfologie di rottura in cedimenti per fatica non facilmente riconducibili a modelli micromeccanici noti. Ciascuna di esse necessita di uno sviluppo di considerazioni particolari e porta a risultati che possono essere considerati dirimenti solo in alcuni casi. Tipiche di queste situazioni sono le fratture per fatica in componenti in acciaio a struttura perlitica o ferritico-perlitica con limitate zone ferritiche o a componenti in lega di alluminio per fonderia, da fusioni di leghe a base di $\mathrm{Al}$ e Si che cedono per fatica a temperatura ambiente. Alcuni esempi e le considerazioni inerenti il riconoscimento di morfologie di rotture per fatica vengono qui di seguito presentate.
\end{abstract}

Parole ChIAVE. Fatica; Frattografia; Striature; Perlite; Leghe Al-Si-Mg; Microstrutture complesse.

\section{INTRODUZIONE}

$\mathrm{D}$ opo la nucleazione delle cricche di fatica a partire da una superficie esterna, quando queste riescono a penetrare nel materiale ad una distanza di alcuni grani dalla superficie, esse tendono a coalescere e quindi a propagare in direzione macroscopicamente normale a quella della tensione principale massima. Si noti che questo stadio, a differenza di quanto avviene nella nucleazione, è governato essenzialmente dalla ampiezza della tensione principale massima in trazione [1]. 
I meccanismi di propagazione in questo stadio sono molteplici; i tre modi più comuni [2] sono:

$\checkmark$ per formazione di striature (materiali duttili);

$\checkmark$ per coalescenza di microvuoti (materiali duttili);

$\checkmark$ per microclivaggio o frattura intergranulare (materiali fragili).

Ovviamente vi possono essere combinazioni di questi, specie se ci si trova di fronte a microstrutture miste. In Fig.1 sono rappresentati schematicamente i primi due stadi della fatica.

La propagazione avviene globalmente in direzione normale a quella della sollecitazione principale massima originata dal carico ciclico applicato (spesso a zig-zag). Nella Fig.1 si è supposto che la propagazione sia transcristallina, cosa usuale in molti materiali, ma in alcuni casi la propagazione può avvenire anche lungo i bordi dei grani (propagazione intercristallina) [2].

Se si osserva la superficie di frattura con un microscopio elettronico a scansione (SEM) è possibile a volte osservare in materiali duttili, come già detto, la presenza di striature (Fig. 2) [3]. Ognuna di queste striature, se la cricca è lunga, rappresenta la crescita della cricca in corrispondenza di un ciclo di sollecitazione.

Molti studi sono stati fatti per comprendere il meccanismo di formazione delle striature [4]. Uno dei modelli qualitativi più diffusi è quello dello "arrotondamento plastico" (plastic blunting process). Il modello è rappresentato nella Fig. 3.

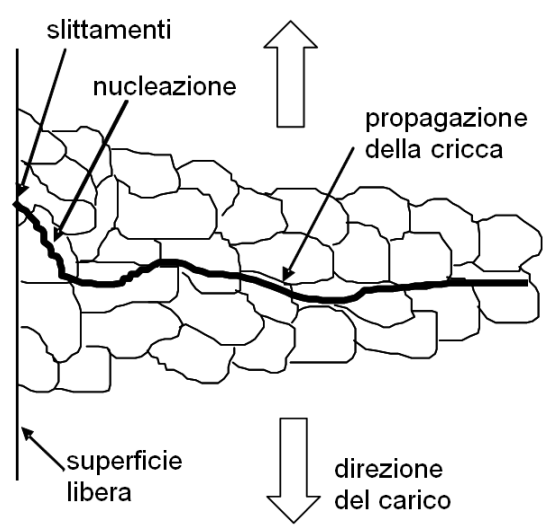

Figura 1: Rappresentazione schematica degli stadi I e II della fatica [1].

Figure 1: I and II fatigue growth stages [1].
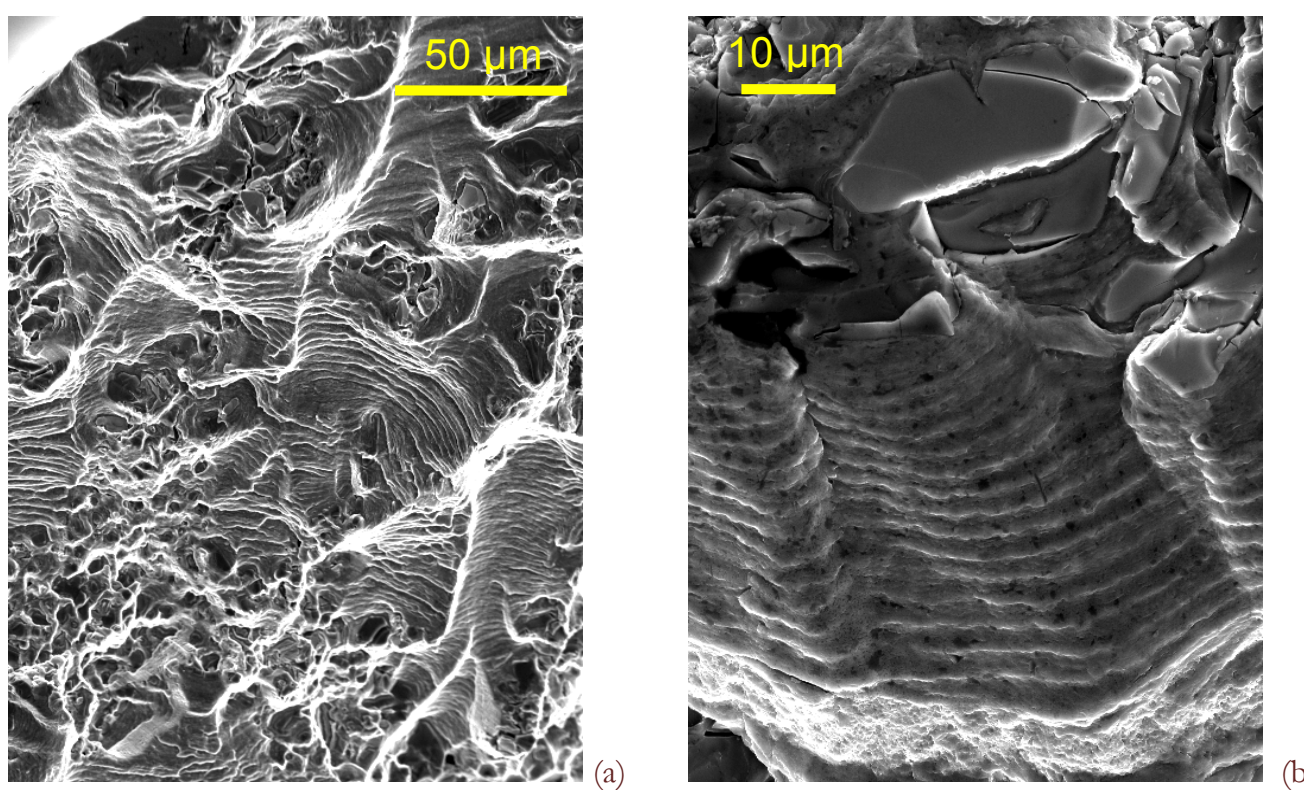

Figura 2: Striature in una lega di alluminio W319-T7 (EN AC-AlSi7Cu3Mg -T7) per fonderia. $\mathrm{R}=-1 ;(\mathrm{a}): \sigma_{\max }=140 \mathrm{MPa}, \mathrm{T}=150{ }^{\circ} \mathrm{C}$; (b): $\sigma_{\max }=100 \mathrm{MPa}, \mathrm{T}=250^{\circ} \mathrm{C}[3]$.

Figure 2: Crack Propagation striations in a W319 - T7 (EN AC-AlSi7Cu3Mg -T7) cast aluminum alloy. R $=-1 ;$ (a): $\sigma_{\max }=140 \mathrm{MPa}, \mathrm{T}$ $=150{ }^{\circ} \mathrm{C} ;(\mathrm{b}): \sigma_{\max }=100 \mathrm{MPa}, \mathrm{T}=250^{\circ} \mathrm{C}[3]$. 

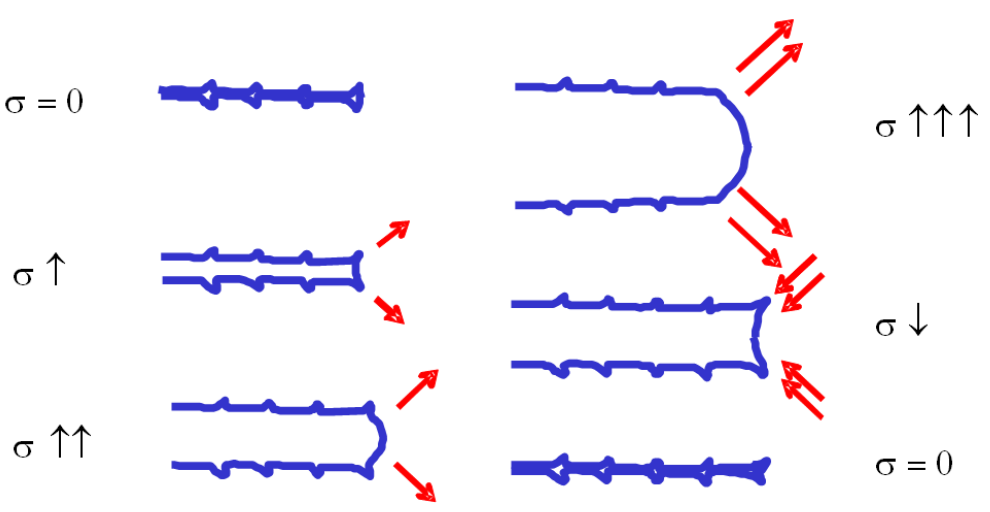

Figura 3: Modello di propagazione delle cricche per arrotondamento plastico [1].

Figure 3: Plastic blunting crack propagation model [1].

Si supponga di avere una cricca già formata a cui viene applicato un ciclo di sollecitazione normalmente alla sua superficie. Anche con tensioni globali basse, le sollecitazioni locali all'apice della cricca sono elevate e superano il limite di snervamento. All'apice della cricca si hanno slittamenti plastici (a volte la formazione di bande di slittamento) che si moltiplicano all'aumentare della sollecitazione esterna, estendendosi a tutta la radice, provocando uno scorrimento globale del materiale ed un arrotondamento dell'apice della cricca. Nella fase di scarico il materiale che ha subito la deformazione plastica non è in grado di tornare alla condizione iniziale e si ha quindi un allungamento della cricca con formazione di una nuova striatura. La successiva riapplicazione di una tensione locale positiva trova un materiale fortemente danneggiato (incrudito) dalle deformazioni plastiche cicliche e una successiva frattura dello stesso materiale per tutto il tratto di materiale danneggiato nei primi momenti di aumento del carico ciclico. Con il seguente ulteriore aumento di tensione si hanno i successivi fenomeni di arrotondamento ed il processo ricomincia. La zona danneggiata dalla applicazione della sollecitazione ciclica è limitata ed è molto meno estesa della zona plasticizzata in seguito al raggiungimento dei valori di $\sigma_{\max }[2]$. Essa prende il nome di "zona plastica ciclica".

La formazione di striature o micro-vuoti nei materiali duttili dipende dal campo di tensioni all'apice della cricca in propagazione e dalla duttilità della lega; per leghe piuttosto duttili come le leghe di alluminio per deformazione plastica è possibile la formazione di striature già a temperatura ambiente; nel caso delle leghe di alluminio per fonderia, meno duttili delle precedenti, si possono avere striature evidenti solo da $150^{\circ} \mathrm{C}$ in su per alti valori della $\sigma_{\max }$ applicata, o da $250{ }^{\circ} \mathrm{C}$ in su per valori più limitati della $\sigma_{\max }$ applicata (Fig.2).

La stessa lega della Fig. 2 a temperatura ambiente non mostra la formazione di evidenti striature nella zona di propagazione di fatica [3]. Nella Fig. 4 è riportata, ad esempio, una zona di frattura per fatica in una lega AlSi7Mg-T6, simile come microstruttura alla lega W319-T7.

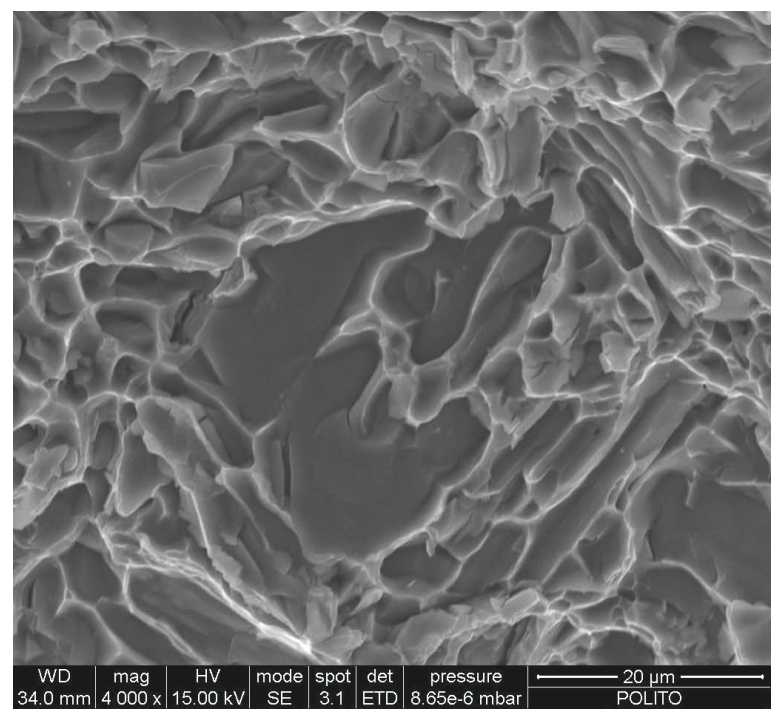

Figura 4: Superficie di frattura per fatica a temperatura ambiente in una lega AlSi7Mg-T6 [1]

Figure 4: Fatigue fracture surface in an AlSi7Mg-T6 alloy at Room Temperature [1]. 
La differenza con la morfologia della frattura riportata in Fig.2 è evidente. Nel primo caso, a causa della temperatura più alta di quella ambiente, la matrice ha sufficiente duttilità per presentare una decoesione con le zone di silicio dure, mentre nel secondo caso la duttilità è insufficiente. Non vi è quindi decoesione matrice metallica/cristalli di silicio, ma solo strizione locale durante la deformazione plastica finale dei legamenti metallici della matrice rimasti fra le cavità generate dalla rottura delle inclusioni di silicio.

È evidente che le microstrutture locali che si possono incontrare durante la propagazione delle rotture per fatica in un componente metallico influenzano pesantemente la morfologia della superficie di frattura, anche in condizioni di duttilità locale, rendendone talvolta molto difficile l'attribuzione esatta del macromeccanismo che la ha causata.

\section{FRATTURE DI FATICA NELLA PERLITE}

$\mathrm{P}$ articolarmente interessante è l'analisi che si può condurre osservando il comportamento della perlite sotto sollecitazioni cicliche. Se si considera la Fig. 5, nella quale è riportato il diagramma relativo alla crescita per fatica di una cricca nella perlite, si può verificare che i valori di $\Delta \mathrm{K}$ per i quali è valida la legge di Paris sono compresi fra 20 e $70 \mathrm{MPa} \sqrt{\mathrm{m}}\left(\Delta \mathrm{K}_{\mathrm{th}} \approx 10 \mathrm{MPa} \sqrt{\mathrm{m}}\right)$.

A questi valori di $\Delta K$, con $R=0,1$ corrispondono valori di $K_{\max }$ compresi fra 22 e $78 \mathrm{MPa} \sqrt{\mathrm{m}}_{\text {. L'estensione della zona }}$ plastificata davanti all'apice della cricca, corrispondente a tali valori di $\mathrm{K}_{\max }$, varia fra $2,5 \cdot 10^{-5} \mathrm{~m}$ e $3,8 \cdot 10^{-3} \mathrm{~m}$ (a seconda che si adottino le formule valide per la sola ferrite in condizioni di tensione piana o per la perlite in condizione di deformazione piana); anche la zona plastica ciclica è più grande dello spessore delle lamelle di ferrite: $10^{-6}-10^{-7} \mathrm{~m}$, a seconda della loro temperatura media di formazione. Ne deriva che, quando la cricca di fatica si avvicina ad una colonia perlitica, Fig. 6, la zona plastificata al suo apice in corrispondenza del $K_{\max }$ applicato è sicuramente superiore allo spessore di una lamella di ferrite e si estende a molte lamelle di ferrite e cementite, provocando la rottura di queste ultime, che hanno una duttilità molto limitata (Fig. 7a,b). [5]

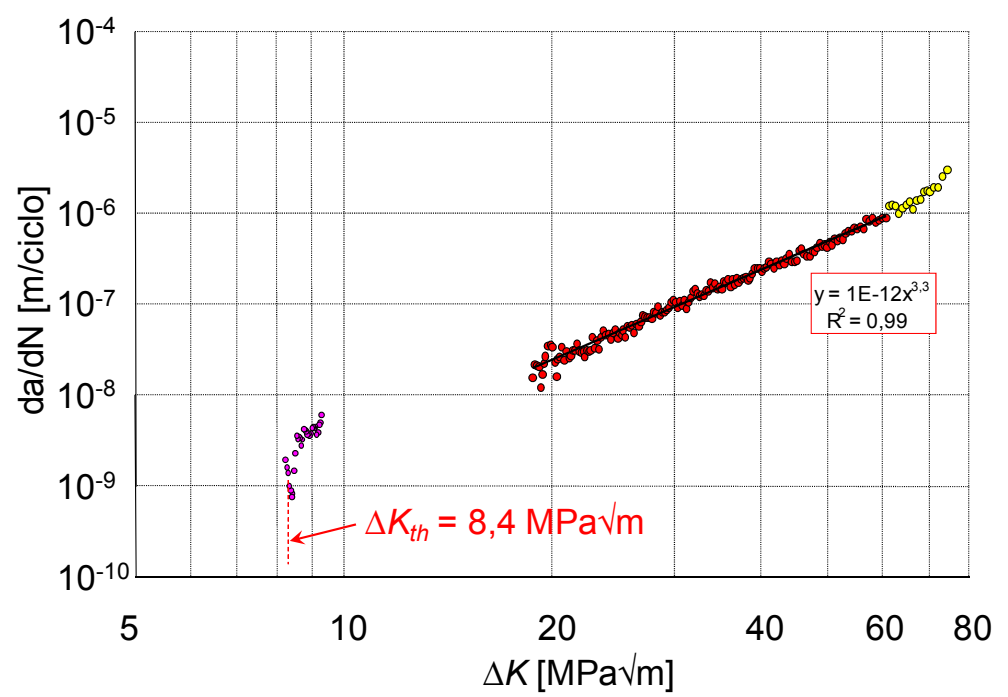

Figura 5: Determinazione sperimentale di curve di propagazione di cricca per una struttura perlitica (a) e del relativo valore di $\Delta K$ di soglia, $\Delta K_{\mathrm{th}}(\mathrm{b})(\mathrm{R}=0,1)[5]$.

Figure 5: Fatigue propagation rate tests for a pearlitic structure (a) and threshold $\Delta K_{\text {th }}$ determination (b) $(\mathrm{R}=0,1)$. [5]

Rimane quindi a resistere davanti alla cricca di fatica in propagazione un pettine di lamelle di ferrite, che la cricca supererà una alla volta. Qualsiasi sia l'angolo con il quale la cricca in propagazione si presenta davanti alla colonia perlitica, lo spessore della lamella di ferrite che si presenta davanti all'apice della cricca per valori bassi di $\Delta \mathrm{K}$ applicato è superiore all'incremento di lunghezza per ciclo $\left(\approx 10^{-8} \mathrm{~m}\right)$. La cricca impiega quindi alcuni cicli per superare la lamella di ferrite che si trova ora in stato di tensione piana (Fig.7c); dopo aver saltato il vuoto corrispondente alla lamella di cementite seguente, già rotta, la cricca si ritrova ad affrontare la rottura della prossima lamella di ferrite.

Solo negli stadi con $\Delta \mathrm{K}$ applicato maggiore di circa $40 \mathrm{MPa} \sqrt{\mathrm{m}}_{\mathrm{m}}$, il valore di da/dN raggiunge e supera il valore dello 
spessore delle lamelle di ferrite, consentendo alla cricca di superare una lamella di ferrite con un solo ciclo. Considerando gli ingrandimenti che si possono efficacemente raggiungere in un microscopio elettronico a scansione, è piuttosto difficile individuare le striature sulla sommità di una lamella di ferrite dopo una rottura di fatica.

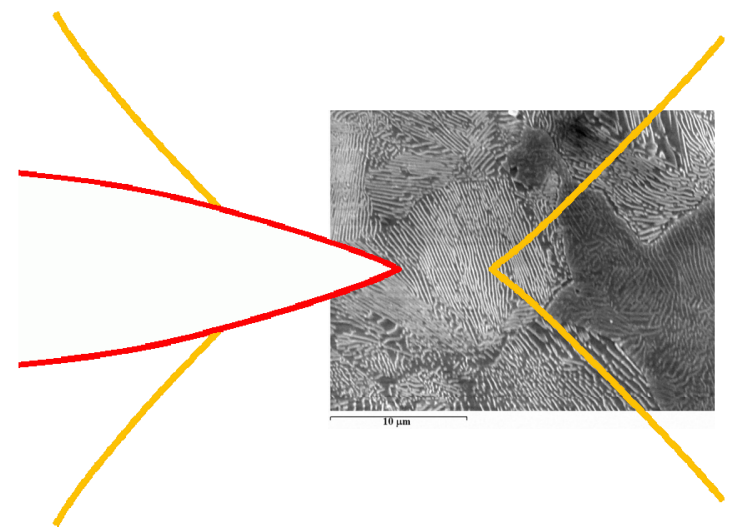

Figura 6: Zona plastificata di fronte all'apice della cricca di fatica propagante attraverso colonie perlitiche a cuore di un blumo di acciaio UNIEN 1.2738, temprato e rinvenuto. Sono presenti anche limitate zone di austenite residua e bainite modificate dal rinvenimento.

Figure 6: Plastic zone in front of the fatigue crack tip at pearlite colonies in the core of a quenched and tempered UNI-EN 1.2738 steel bloom. Small areas of residual austenite and bainite as modified by tempering are also present.

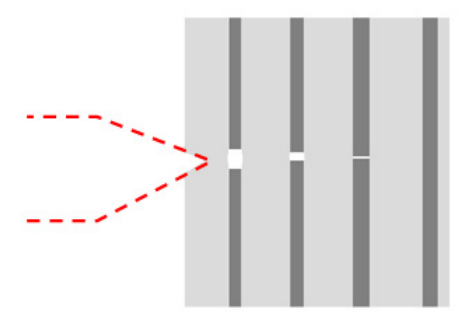

(a)

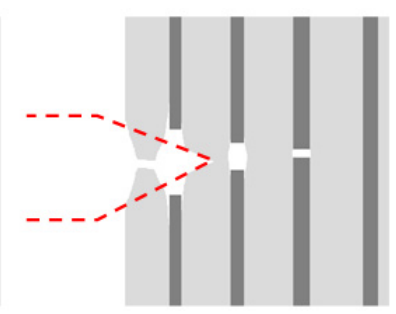

(b)

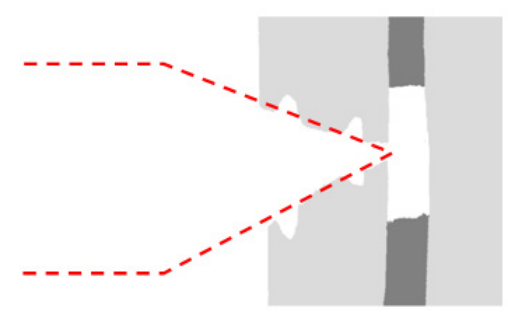

(c)

Figura 7: Schema di propagazione di una cricca di fatica in una colonia perlitica $(\mathrm{a}, \mathrm{b})$; formazione di striature alla sommità di una lamella di ferrite rotta (c).

Figure 7: Fatigue crack propagation model in a pearlitic colony $(\mathrm{a}, \mathrm{b})$; striation formation at the top of a broken ferrite lamella (c).

In Fig.8 [6] è riportata la frattura per fatica in corrispondenza di valori di $\Delta \mathrm{K}$ medi per una zona perlitica di cuore di una barra di $90 \mathrm{~mm}$ di diametro, fabbricata con acciaio 34CrMo4 legato con boro, temprato e rinvenuto. Si vedono i pettini di lamelle di ferrite rotte durante l'avanzamento della cricca di fatica e rare striature in zone limitate della sommità delle lamelle.

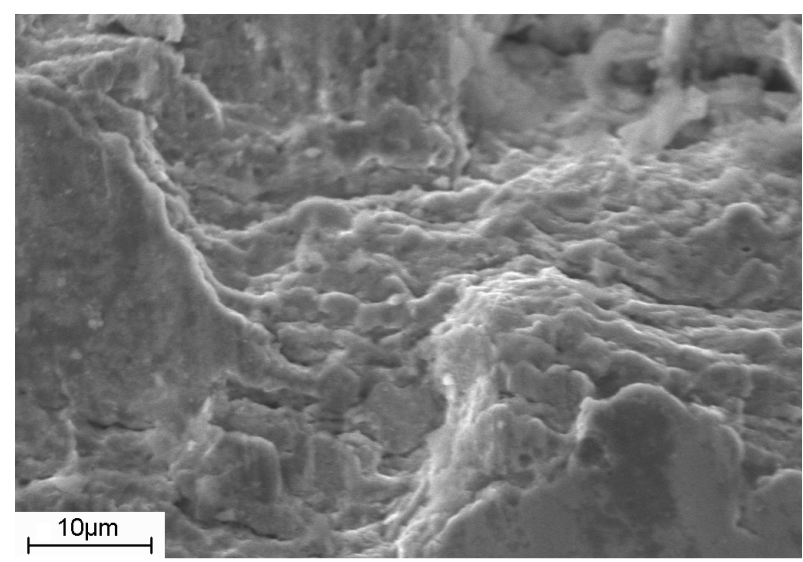

Figura 8: Rottura a fatica di zone perlitiche in un acciaio UNI 34CrMo4 legato con B, temprato e rinvenuto. Regione a cuore di una barra di diametro $90 \mathrm{~mm}$. Microstruttura prevalente a cuore ferritico-perlitica [6].

Figura 8: Fatigue rupture of pearlitic areas at core of a quenched and tempered, B alloyed, 34CrMo4 steel bar, with a $90 \mathrm{~mm}$ dia. bar. Ferriticpearlitic microsructure prevailing at core [6]. 


\section{FRATTURE DI FATICA NELLE LEGHE AL-Si IPOEUTETTICHE}

S

i hanno ora gli strumenti per comprendere la genesi della frattura per fatica riportata in Fig. 4. La lega, come già detto, è classificabile come AlSi7Mg-T6 (A356.0) e, secondo la norma UNI EN 1706:2010, come lega EN AC 42100 colata in conchiglia. Le caratteristiche meccaniche minime di tale lega riportate nelle tabelle UNI-EN 1706:2010 sono: $\mathrm{R}_{\mathrm{m}}=290 \mathrm{MPa}, \mathrm{R}_{\mathrm{p} 0,2}=210 \mathrm{MPa}, A=4 \%, H B=90$. Dal complesso dei risultati riportati in letteratura si può attribuire a tale lega una tenacità a frattura $K_{\mathrm{Ic}} \approx 27 \mathrm{MPa} \sqrt{\mathrm{m}}$ ed un limite di fatica a $5 \cdot 10^{7}$ cicli di $90-100 \mathrm{MPa}$. Le norme americane relative alla lega A 356.0 colata in conchiglia indicano come valori minimi delle caratteristiche meccaniche: $R_{\mathrm{m}}=260 \mathrm{MPa}, R_{\mathrm{p} 0,2}=185 \mathrm{MPa}, A=5 \%, H B=80$. I valori minimi delle caratteristiche meccaniche incontrati in letteratura [7] sono $R_{\mathrm{m}}=190 \mathrm{MPa}, R_{\mathrm{p} 0,2}=155 \mathrm{MPa}, A=4 \%$, e $K_{\mathrm{Ic}} \approx 21 \mathrm{MPa} \sqrt{\mathrm{m}}_{\mathrm{m}}$. Assumendo qualsiasi coppia di valori $K_{\mathrm{Ic}}$ ed $R_{\mathrm{p} 0,2}$, le dimensioni delle zone plastiche statiche sono dell'ordine del $\mathrm{mm}$; per quanto più piccole, le zone plastiche cicliche comprendono molte inclusioni di silicio (Fig. 9,10).

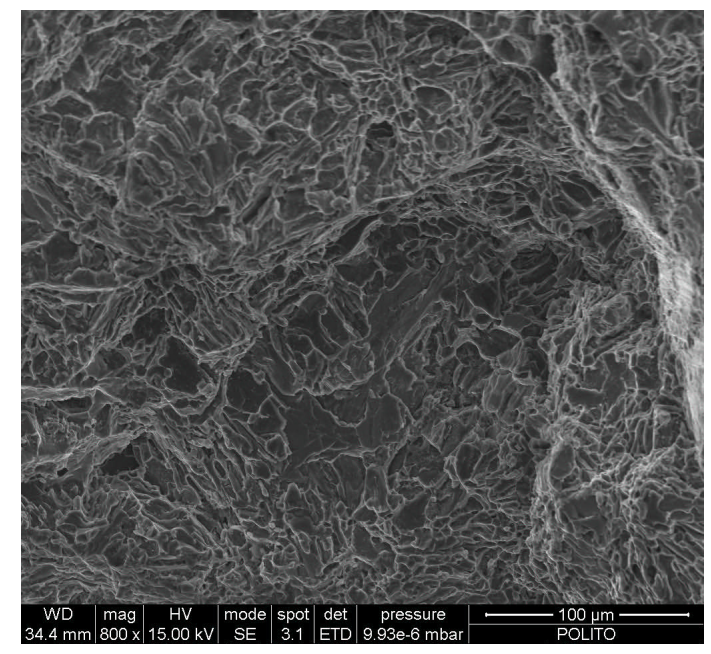

Figura 9: Particolare di una superficie di rottura a fatica in una lega AlSi7Mg-T6.

Figure 9: Detail of a fatigue rupture surface in an AlSi7Mg-T6 alloy.

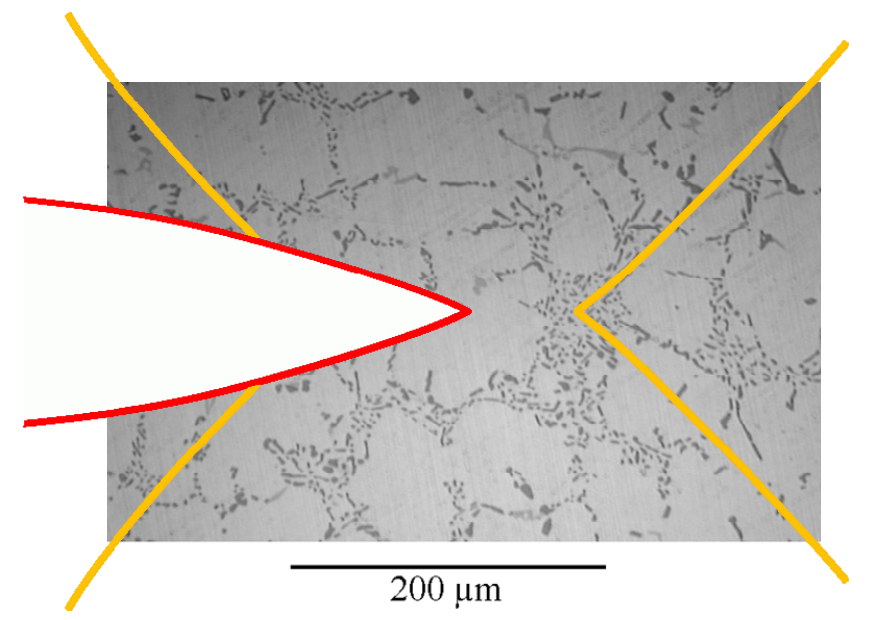

Figura 10: Zona plastica di fronte all'apice della cricca di fatica propagantesi in una lega AlSi7Mg-T6.

Figure 10: Plastic zone in front of the fatigue crack tip growing in an AlSi7Mg-T6 alloy.

Anche in questo caso si deve assumere che davanti all'apice della cricca, che nella sua propagazione segue le zone eutettiche, ricche di inclusioni di silicio, si abbia subito la rottura di tali inclusioni e che rimanga a resistere un insieme di legamenti costituiti prevalentemente da lega Al-Si con circa l'1,5\% di Si. Tali legamenti hanno spessore dell'ordine di $5 \cdot 10^{-6} \mathrm{~m}$. 
Riprendendo la zona di frattura riportata in Fig. 4, la sovrapposizione della zona plastificata porta alla Fig. 11. E' chiaramente da concludere che l'arrivo della zona plastificata ciclica porta prima di tutto alla rottura delle inclusioni di silicio, lasciando a resistere alla propagazione definitiva della cricca un reticolo di legamenti di matrice.

L'espressione numerica della legge di Paris relativa a tale tipo di lega può essere assunta simile alla seguente:

$$
d a / d N=10^{-10 \cdot \Delta K^{4},}
$$

$\operatorname{con} \Delta K_{\text {th }}$ dell'ordine di $2 \mathrm{MPa} \sqrt{\mathrm{m}}_{\mathrm{m}}$ [8, 9]

Ipotizzando $\mathrm{R}=0$, a valori di $K_{\max }$ dell'ordine di $15 \mathrm{MPa} \sqrt{\mathrm{m}}$ corrispondono valori di $d a / d N \approx 5 \cdot 10^{-6} \mathrm{~m}$. Quindi, da questo $K_{\max }$ in poi, ad ogni ciclo si ha la rottura intera di un legamento e non si ha la possibilità di vedere striature su di esso. Per valori di $K_{\max }$ inferiori a 15 vi sarebbe la possibilità di osservare striature nella frattura dei legamenti.

Ad esempio, per valori di $K_{\max }$ compresi fra 5 e $6 \mathrm{MPa} \sqrt{\mathrm{m}}_{\mathrm{m}}$ si dovrebbero avere $d a / d \mathrm{~N}$ di poco superiori a $10^{-8} \mathrm{~m}$, il che porterebbe a poter vedere una striatura con un ingrandimento fra 5.000 e 10.000 volte. A tali ingrandimenti solo i migliori microscopi elettronici a scansione offrono una visione sufficientemente nitida (Fig. 12).

Nella Fig. 12 si notano sulla sommità dei legamenti rotti, presenti fra le particelle di silicio (scure), deboli morfologie di frattura che possono fare pensare a striature.

$\mathrm{Si}$ deve in ogni caso concludere che il riconoscimento frattografico di una frattura di fatica in una lega Al-Si-Mg risulta indubitabilmente impegnativo.

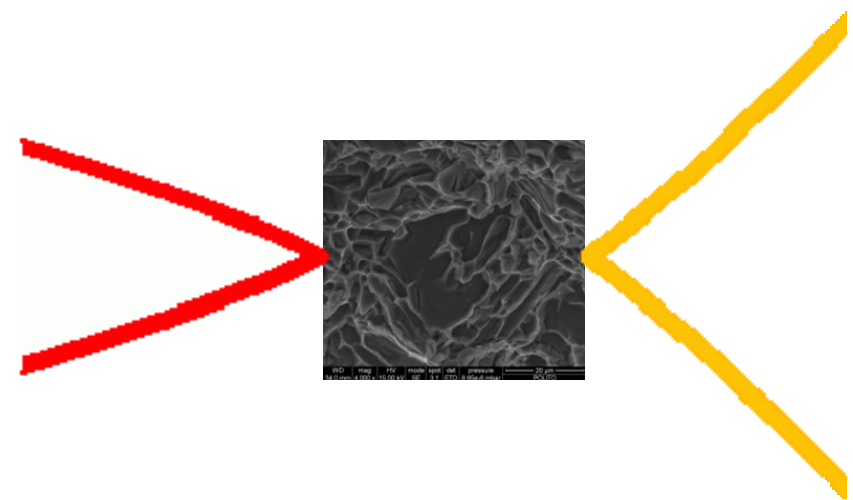

Figura 11: Sovrapposizione della zona plastificata di fronte all'apice della cricca di fatica in propagazione con la zona di frattura riportata in Fig. 4.

Figure 11: Overposition of the plastic zone at the tip of a fatigue crack with the fracture area of Fig. 4.

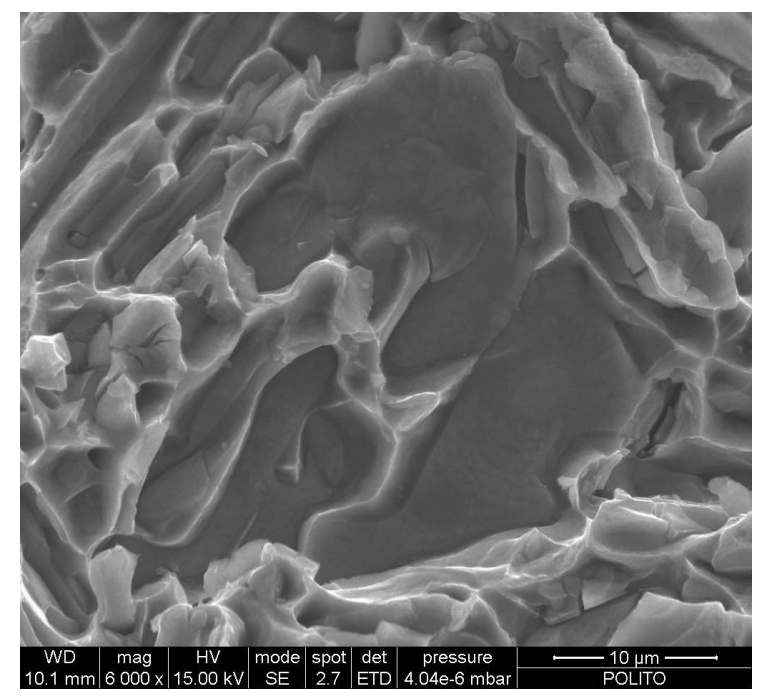

Figura 12: Ingrandimento della zona di frattura riportata in Fig. 4. Si noti che il campione è stato ruotato, rispetto alla visione di Fig. 4, di $180^{\circ}$ attorno ad un asse perpendicolare alla superficie di frattura, per ottenere una migliore illuminazione e contrasto.

Figure 12: Enlargement of the fracture area of Fig. 4. Please note that the specimen has been here rotated by a $180^{\circ}$ angle around the axis normal to the overall rupture plane to achieve better light and contrast. 


\section{CONCLUSIONI}

I stato dimostrato che il riconoscimento frattografico delle rotture di fatica in leghe metalliche con microstrutture complesse nelle quali intervengono più fasi o più costituenti metallografici è particolarmente impegnativo. I micro meccanismi ipotizzati devono essere provati tenendo presente contemporaneamente le formule per l'estensione della zona plastificata all'apice della cricca in propagazione e le equazioni rappresentative della legge di Paris per le particolari leghe oggetto di indagine.

Sono stati riportati esempi di applicazione di quanto sopra affermato alla propagazione per fatica in microstrutture perlitiche ed in microstrutture eutettiche presenti in una lega Al-Si-Mg ipoeutettica.

\section{BIBLIOGRAFIA}

[1] D. Firrao, M. Rossetto, In: Tenacità e resistenza a fatica delle leghe metalliche, curatori: R. Donnini, R. Montanari, M. Vedani. AIM - Associazione Italiana di Metallurgia, Milano, (2011) 31.

[2] H.O. Fuchs, R.I. Stephens, Metal fatigue in engineering, John Wiley, New York (1980).

[3] A. Shyam, J.E. Allison, J.W. Jones, risultati non pubblicati.

[4] A.J. McEvily, H. Matsunaga, Scientia Iranica, Transaction B: Mechanical Engineering, 17 (2010) 75.

[5] D. Firrao, P. Matteis, Frattura ed Integrità Strutturale, 18 (2011) 54-68.

[6] D. Firrao, P. Matteis, P. Russo Spena, G.M.M. Mortarino, Int. J. of Fatigue, 32 (2010) 864.

[7] V.S. Zolotorevsky, N. Belov, M.V. Glazov, Casting aluminum alloys, Elsevier, Amsterdam, (2007) 304.

[8] M.J. Couper, A.E. Neeson, J.R. Griffiths, Fatigue \& Fracture of Engineering Materials \& Structures, 13 (1990) 213.

[9] M.J. Caton, J.W. Jones, J.E. Allison, In: Fatigue crack growth thresholds, endurance limits, and design, ASTM-STP 1372, curatori: J.C. Newman, R.S. Piascik, American Society for Testing and Materials, West Conshohocken, PA, (2000). 